\title{
A FUSION-DRIVEN GAS CORE NUCLEAR ROCKET
}

\author{
T. Kammash \\ T. Godfroy \\ Department of Nuclear Engineering and Radiological Sciences \\ The University of Michigan \\ Ann Arbor, MI 48109 USA
}

(313) 764-0205

\begin{abstract}
A magnetic confinement scheme is investigated as a potential propulsion device in which thrust is generated by a propellant heated by radiation emanating from a fusion plasma. The device in question is the gasdynamic mirror (GDM) machine in which a hot dense plasma is confined long enough to generate fusion energy while allowing a certain fraction of its charged particle population to go through one end to a direct converter. The energy of these particles is converted into electric power which is recirculated to sustain the steady state operation of the system. The injected power heats the plasma to thermonuclear temperatures where the resulting fusion energy appears a charged particle power, neutron power, and radiated power in the form of bremsstrahlung and synchrotron radiation. The neutron power can be converted through a thermal converter to electric power that can be combined with the direct converter power before being fed into the injector. The radiated power, on the other hand, can be used to heat a hydrogen propellant introduced into the system at a specified pressure and mass flow rate. This propellant can be pre-heated by regeneratively cooling the (mirror) nozzle or other components of the system if feasible, or by an electrothermal unit powered by portions of the recirculated power. Using a simple heat transfer model that ignores the heat flux to the wall, and assuming total absorption of radiation energy by the propellant it is shown that such a gas core rocket is capable of producing tens of kilonewtons of thrust and several thousands of seconds of specific impuise. It is also shown that the familiar Kelvin-Helmholtz instability which arises from the relative motion of the neutral hydrogen to the ionized fuel is not likely to occur in this system due to the presence of the confining magnetic field.
\end{abstract}

\section{INTRODUCTION}

We examine in this paper a nuclear propulsion system which utilizes fusion reactions to generate energy while allowing a certain segment of its charged particle population to escape to generate thrust. Because the ejected particles are isotopes of hydrogen and their mass is small, the thrust generated may be viewed as modest when compared to the very large specific impulse produced by the system. Thrust enhancement can, however, be achieved with the aid of a hydrogen propellant which may be pre-heated by regenerative cooling of the nozzle, or other components, and further heated by the radiation emitted by the fusion plasma. This can lead to a significant increase in the thrust but at a modest specific impulse, a situation that might be called for in certain space missions.

The device in question is the gasdynamic mirror (GDM) fusion propulsion concept shown in Fig. 1. It makes use of a high density plasma in a magnetic mirror geometry with a collision mean free path, $\lambda$ much shorter than its length, $L$, i.e. :

$$
\frac{\lambda}{R} \ll L \text {, }
$$

where $R$ is the mirror ratio seen by the plasma and related to the vacuum mirror ratio $R_{o}$ by : 
This quantity which represents the ratio of the strength of the mirror magnetic field to that of the central field reflects the ability of the device in containing the fusion plasma. Since such a plasma is diamagnetic, in that it attempts to exclude the magnetic field, the quantity $\beta$ which connects the two mirror ratios represents the ratio of the plasma pressure $(n k T)$ to that of the vacuum magnetic field pressure. It is in effect a measure of the efficiency of this magnetic field in confining the plasma, and since it is proportional to the plasma density, $n$, it is also a measure of the fusion power density supportable by the system.

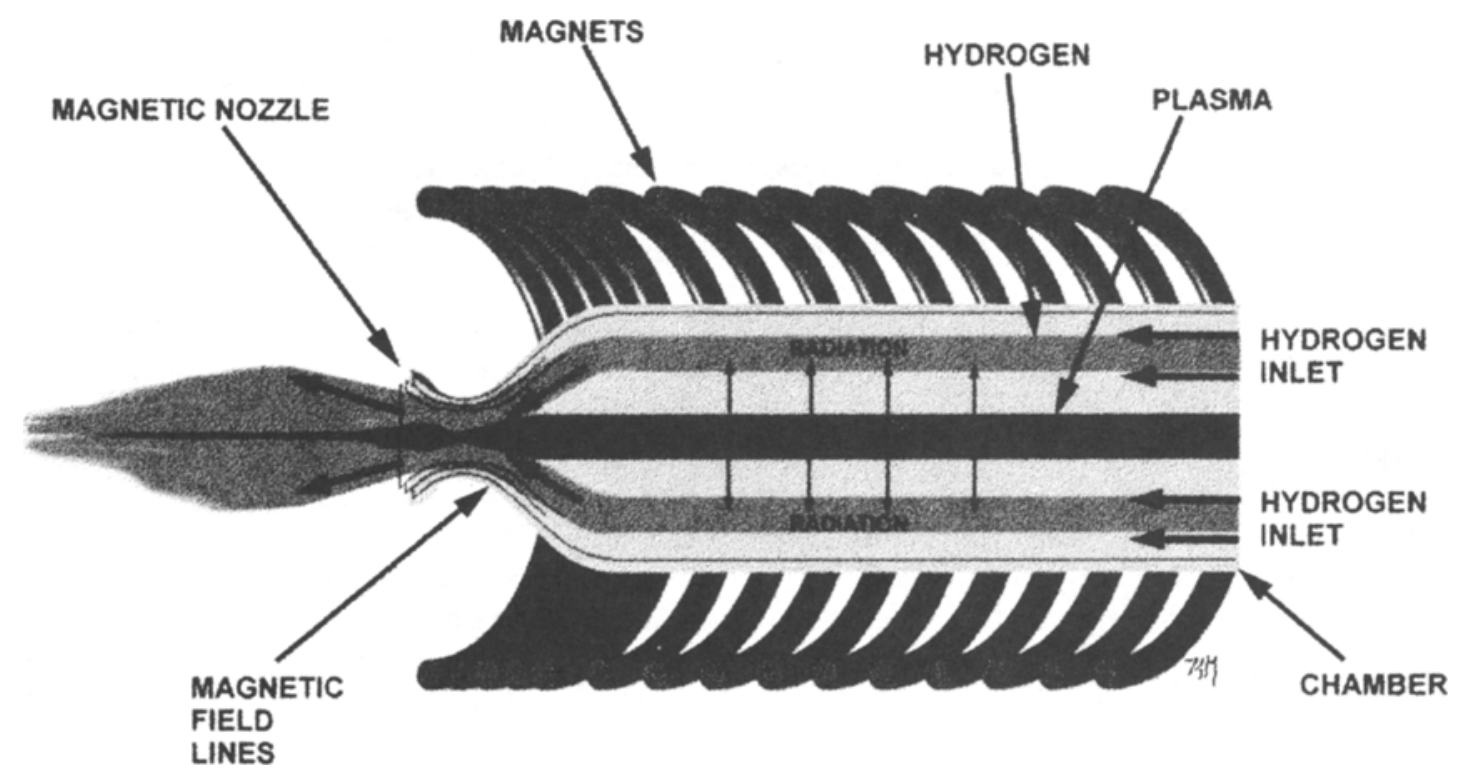

FIGURE 1. Thrust Enhanced Gasdynamic Mirror Fusion Propulsion Engine

In a propulsion system we envisage the mirror reactor as forming along with the injector the GDM engine which generates the energy from the fusion reactions while allowing a fraction of its population to escape from one end, which also serves as a magnetic nozzle, to provide thrust. As illustrated in Fig. 2 the injector provides the power needed to heat the plasma to thermonuclear temperatures, and upon ignition an energy multiplication given by $Q$ takes place in the reactor. With a deuterium-tritium (DT) fuel cycle the reaction products are neutrons with power, $P_{n}$, and charged particles (including alpha particles) with power $P_{c}$, along with radiation power, $P_{r}$, consisting of bremsstrahlung, $P_{B}$, and synchrotron radiation, $P_{s}$. The neutron and radiation power are processed by a thermal converter at an efficiency $\eta_{t}$, while a fraction $f$ of the charged particles travel through one mirror to a direct converter where their power is converted to electric power at an efficiency $\eta_{D}$. We have added to the power flow diagram a hydrogen (or other suitable propellant) pre-heat component such as an electrothermal unit $\left(P_{H}\right)$ that can be utilized in a gas-core mode of operation that calls for the use of an auxiliary propellant to enhance the thrust. If we set the gross electric power, $P_{G}$, produced by the system exactly equal to the sum of the power to the injector. $P_{i n}$, and $P_{H}$ we obtain the critical $Q$-value i.e. that which is required for the system to be self-supporting, namely :

$$
\mathrm{Q}_{\mathrm{c}}=\frac{1-f \eta_{i} \eta_{D}}{f \eta_{i} \eta D-\frac{P_{H}}{P_{f}}-\frac{\left(P_{n}+P_{r}\right)}{P_{f}} \eta_{i}\left(f \eta D-\eta_{t}\right)}
$$


where $\eta_{t}$ is the injector efficiency usually set equal to unity and $P_{f}$ the fusion power. For a symmetric mirror i.e. $f=$ 1/2 with $P_{H}=0$ the critical $Q$-value for the reactor with $\eta_{t}=1, \eta_{t}=0.45$ and $\eta_{D}=0.90$ is 1.222 which is quite modest and easily achievable. It might be noted that this $Q$-value is much smaller than one required for a terrestrial power reactor. It can therefore be argued that the conditions for a propulsion device are much less stringent than those for a power reactor, making GDM an especially attractive candidate for space propulsion. It should also be added that $f \neq 1 / 2$ i.e. an asymmetric mirror may provide a more optimum performance when electric power production is balanced against jet power (Kammash 1997).

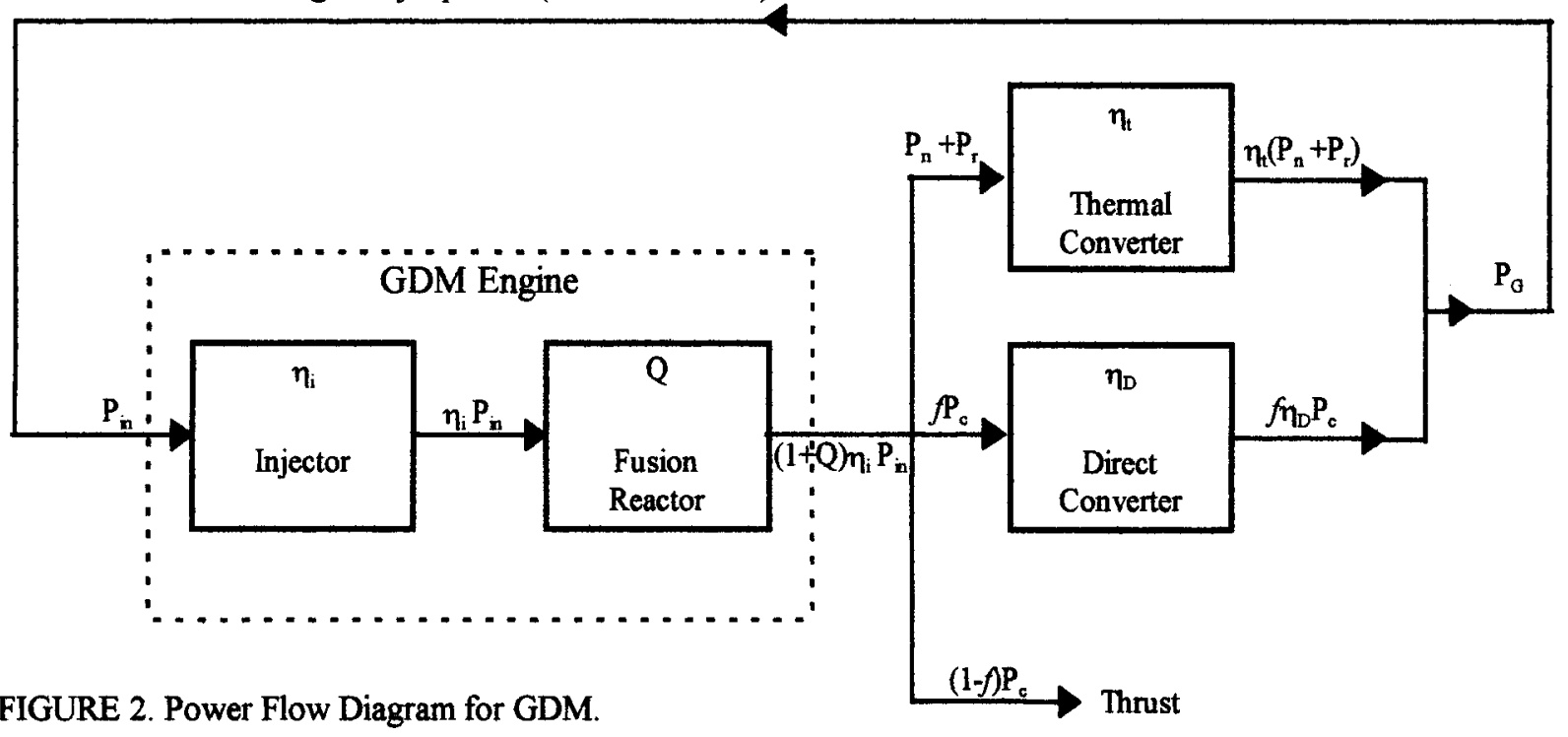

The operating condition represented by Eq. (1) makes the plasma in GDM behave like a continuous medium - a fluid. Under these circumstances the escape of the plasma from the device is analogous to the flow of a gas into a vacuum from a vessel with a hole. The confinement time is obtained from gasdynamic laws and is given by (Mirnov 1979) :

$$
\tau=\frac{R L}{v_{t h}},
$$

where $v_{t h}$ is the particles thermal velocity. It can be utilized in establishing the steady state operation of the reactor which is governed by the following mass and energy conservation equations, respectively:

$$
\begin{aligned}
& S-\frac{n}{\tau}-\frac{n^{2}}{2}\langle\sigma v\rangle=0, \\
& S E_{i n}+\frac{n^{2}}{4}\langle\sigma\rangle E_{L}-\frac{n E_{L}}{\tau}-P_{B}-P_{S}=0,
\end{aligned}
$$

In these equations $S$ is the rate of injection of the fuel ions per unit volume, $\langle\sigma v\rangle$ the velocity-averaged fusion reaction cross section. $E_{\text {in }}$ the energy of injected particles and $E_{c}$ the portion of fusion energy that remains in the plasma, which in the case of D-T fuel, is equal to the alpha particle energy of $3.5 \mathrm{MeV}$. The quantity $E_{L}$ represents the mean energy of escaping ions which for a Maxwellian distribution can be shown to be equal to twice the temperature or $2 T$ (Kammash 1995). The remaining terms in Eq. (6), namely $P_{B}$ and $P_{s}$ are the radiation terms introduced earlier. Instead of specifying the injection energy, $E_{\text {in }}$, we find it convenient to express it in terms of the $Q$-value discussed previously or : 


$$
E_{t n}=\frac{E_{L}}{1+\frac{Q}{P_{f}}\left[P_{r}+\frac{n^{2}}{4}\langle\sigma v\rangle\left(2 E_{L}+E_{C}\right)\right]} \text {, }
$$

We recall from' Fig. 2 that $Q$ is the ratio of fusion power to injected power or $P_{f} / S E_{i n}$, and in the absence of fusion energy production and negligible radiation. $E_{i n}$ approaches the escape energy $E_{L}$ in a steady state system as it should.

\section{THE GAS CORE FUSION ROCKET}

In the conventional open cycle gas core nuclear rocket (GCR) energy is produced by a fissioning plasma such as uranium that allows the core to radiate like a black body (Ragsdale 1971). By injecting a hydrogen propellant that flows around the core and hydrodynamically contain it, propulsion is produced by the heated, seeded hydrogen as it exits through a nozzle. It is noted that in such a system the transparency of the uranium plasma and the hot hydrogen allows up to $10 \%$ of the reactor power to appear as radiation that strikes the reactor wall. It is the ability to remove this energy either by means of an external radiator or regeneratively using the hydrogen propellant that determines the maximum power output and achievable specific impulse for GCR engines. It is also noted that due to the relative motion between the propellant and the core the Kelvin-Helmholtz instability (Chandrasekhar 1961) can arise and seriously impact the performance of the system. Such an instability is believed to be amenable to stabilization with the aid of externally applied magnetic field (Chandrasekhar 1961). In view of these facts it has been shown that a specific impulse of about 1000-2000 seconds can be expected from the fission gas core nuclear rocket albeit at a sizable thrust (Poston 1996).

By contrast, the fusion gas core nuclear rocket, illustrated in Fig. 1, has the ability to produce comparable or perhaps better propulsion performance without encountering some of the serious confinement and stability problems. On the one hand, one can operate the system without the auxiliary hydrogen propellant and rely on the fusion plasma to provide the specific impulse and thrust. These performance parameters can be obtained by solving Eqs. (5) and (6) and one illustrative example for such a system is shown in Table 1 for two fusion fuel cycles, a DT and a D-He.

TABLE 1. GDM Plasma and Performance Parameters.

\begin{tabular}{|l|c|c|}
\hline \multicolumn{1}{|c|}{ Parameter } & DT & D-He $^{3}$ \\
\hline Plasma density $\left(\mathrm{cm}^{-3}\right)$ & $1.0 \times 10^{16}$ & $1.0 \times 10^{16}$ \\
\hline Plasma temperature $(\mathrm{keV})$ & 10 & 60 \\
\hline Plasma radius $(\mathrm{cm})$ & 5 & 5 \\
\hline Plasma length $(\mathrm{m})$ & 44 & 1297 \\
\hline Central magnetic field $(\mathrm{T})$ & 9.21 & 24.73 \\
\hline Thrust $\mathrm{F}(\mathrm{N})$ & $2.512 \times 10^{3}$ & $1.437 \times 10^{4}$ \\
\hline Thrust power $(\mathrm{MW})$ & $1.351 \times 10^{3}$ & $1.894 \times 10^{4}$ \\
\hline Bremsstrahlung power $(\mathrm{MW})$ & 58.17 & $1.703 \times 10^{4}$ \\
\hline Synchrotron power $(\mathrm{MW})$ & 18.94 & $4.25 \times 10^{4}$ \\
\hline Engine mass $(\mathrm{Mg})$ & 101 & 3015 \\
\hline Total vehicle mass $(\mathrm{Mg})$ & 422 & 4434 \\
\hline Specific power $(\mathrm{kw} / \mathrm{kg})$ & 13.40 & 6.28 \\
\hline Specific impulse $\mathrm{I}_{\mathrm{p}}(\mathrm{s})$ & $1.268 \times 10^{5}$ & $3.106 \times 10^{5}$ \\
\hline Mars round trip $\tau_{\mathrm{RT}}(\mathrm{d})$ & 169 & 228 \\
\hline
\end{tabular}

In calculating the Mars mission travel time we utilized a constant thrust, constant $I_{s p}$, continuous burn acceleration/deceleration trajectory profile, and used the linear distance $l$ from Earth to Mars that corresponds to the 
configuration when the Earth lies between the sun and Mars (approximately every 26 months). The $\tau_{R T}$ in this case can be expressed by (Kammash 1994)

$$
\tau_{R T}=\frac{4 l}{g I_{s p}}+4 \sqrt{\frac{l m_{f}}{F}},
$$

where $m_{f}$ is the final (dry) mass of the vehicle. The above equation neglects the effects of gravity of the planets involved as well as that of the sun; it also ignores the change in the Earth's position during the flight.

Table 1 reveals, among other things, that GDM where the fuel and the propellant are the same produces very high specific impulse at perhaps a modest thrust. By converting it to an open cycle gas core fusion rocket its propulsive performance may be altered to address certain missions where higher thrust at smaller specific impulse may be required. We accomplish this by injecting hydrogen at high pressure into the chamber as illustrated in Fig. 1. This hydrogen may be pre-heated through regenerative cooling of the nozzle (or other components) in the operation described above, and if that is not adequate a pre-heat unit (e.g. an electrothermal unit) as displayed in Fig. 2 may be used to perform that function. Once inside the reactor chamber the hydrogen will be further heated by the radiation emanating from the plasma which add up, in the case of DT, to about $77 \mathrm{MW}$ as displayed in Table 1. Because it operates at a much higher temperature, the radiation power in the case of $\mathrm{D}-\mathrm{He}^{3}$ fueled reactor is several orders of magnitude higher and can, therefore, be viewed as especially suitable for operation as an open cycle gas core rocket. In both instances, however, the plasma is transparent to this radiation, which for the conditions under consideration has an absorption mean free path much larger that the dimensions of the core. The (seeded) hydrogen is also assumed to be totally opaque to this radiation, a condition that can easily by revoked in a more rigorous analysis of the problem.

If we ignore the emissivity of the hydrogen gas, and neglect heat transfer in the direction of motion, as is usually done for heat conduction in moving fluids, we can write the appropriate energy balance equation as (Diessler 1994) :

$$
\rho C_{p} u \frac{d T_{H}}{d x}=P_{r}
$$

where $P_{r}$ is the radiated power per unit volume or the heat source, $d T_{H} / d x$ is the change in the hydrogen temperature in the direction of flow, $C_{p}$ the specific heat, $\rho$ the density and $u$ the axial flow velocity. The above equation can be rewritten as :

$$
C_{p} \frac{d T_{H}}{d t}=\frac{P_{r}}{\rho}
$$

which upon integration, assuming constant $\rho$ and $C_{p}$, becomes :

$$
T_{H}=T_{H_{\circ}}+\tau_{H} \frac{P_{r}}{\rho C_{p}} \text {, }
$$

where $T_{H_{\mathrm{o}}}$ is the hydrogen inlet temperature, and $\tau_{H}$ the hydrogen residence time given by :

$$
\tau_{H}=\frac{L}{u} \sim \frac{L}{\sqrt{T_{H}}}
$$


We apply this simplified analysis to the GDM gas core case and obtain the results shown in Table 2 for an inlet temperature of $3000 \mathrm{~K}$ and the two fuel cycles used in Table 1.

TABLE 2. Parameters for An Open Cycle Gas Core Fusion Propulsion System.

\begin{tabular}{|l|c|c|}
\hline \multicolumn{1}{|c|}{ Parameter } & DT & D-He $^{3}$ \\
\hline Radiative power $(\mathrm{MW})$ & 77 & $59 \times 10^{3}$ \\
\hline Hydrogen flow rate $(\mathrm{kg} / \mathrm{s})$ & 3.00 & 270.00 \\
\hline Inlet temperature $(\mathrm{K})$ & 3000 & 3000 \\
\hline Hydrogen layer $(\mathrm{cm})$ & 5 & 5 \\
\hline Exit temperature $(\mathrm{k})$ & 8313 & 14,218 \\
\hline Pre-heat power $(\mathrm{MW})$ & $1.746 \times 10^{2}$ & $10^{4}$ \\
\hline Effective thrust $(\mathrm{kN})$ & $32.53(2.512)$ & $3.607 \times 10^{3}(14.37)$ \\
\hline Effective Isp $(\mathrm{s})$ & $1.11 \times 10^{3}\left(1.268 \times 10^{5}\right)$ & $1.36 \times 10^{3}\left(3.106 \times 10^{5}\right)$ \\
\hline Mars trip time $\tau_{\mathrm{RT}}(\mathrm{d})$ & $383(169)$ & $284(228)$ \\
\hline
\end{tabular}

The quantities in parentheses for the last three parameters in Table 2 give the values (shown in Table 1) for which no hydrogen propellant is employed. The significant enhancement in thrust is evident for both fuel cycles although it is much more dramatic in the $\mathrm{D}-\mathrm{He}^{3}$ case. Since the specific impulse is dominated by the hydrogen the drop in this parameter is also evident for both cases, although it is more than made up for by the increase in thrust in the D-He ${ }^{3}$ case. This is reflected in the significant reduction in travel time. The opposite is however true in the D-T case and that may be attributed to the lower operating plasma temperature and the correspondingly small radiative power. It should also be noted that the hydrogen mass flow rates were selected to ensure the integrity of the wall while guarding against triggering the Kelvin-Helmholtz instability which arises when one fluid (the propellant) flows past another (the plasma core) in the presence of a gravitational force. The hydrogen in the present case is introduced at a sufficiently high pressure that makes its density comparable or larger than that of the hot plasma. When that fact is coupled to the flow velocity noted earlier it is possible that short wave length oscillations can become unstable leading to localized turbulence. Such instability can, however, be stabilized by placing the system in an axial magnetic field with a magnitude of about 0.2 tesla, well below the central field value of $9.2 \mathrm{~T}$. Therefore such a situation is not likely to occur in the GDM gas core rocket because of the tension the magnetic field exerts at the boundary between the plasma and the hydrogen, which at these operating temperatures remains unionized and almost oblivious to the presence of the confining magnetic field.

Although hydrogen appears to be a suitable propellant we have examined others for the purpose of identifying the most desirable ones from the point view of propulsion. Although they are of different molecular masses and thermodynamic properties, we used a common mass flow rate to assess their performance. The results are displayed in Fig. 3 where we note the best thrust and specific impulse is provided by hydrogen and helium, and close behind is lithium. The latter is especially important for two reasons: 1) it is a breeder of tritium upon absorption of the fusion neutrons, and 2) it can serve as an appropriate working fluid for a thermal converter that employs, for example, the Brayton Cycle. For these reasons and for the additional advantage of providing protection to the reactor chamber wall from the high heat flux, one might choose lithium as the propellant for the GDM fusion gas core rocket. 


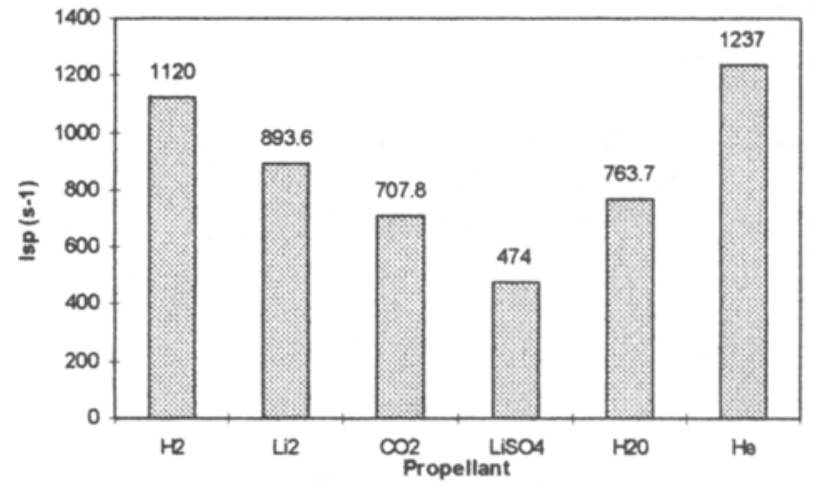

(a) Thrust at $3 \mathrm{~kg} / \mathrm{s}$ Mass Flow Rate.

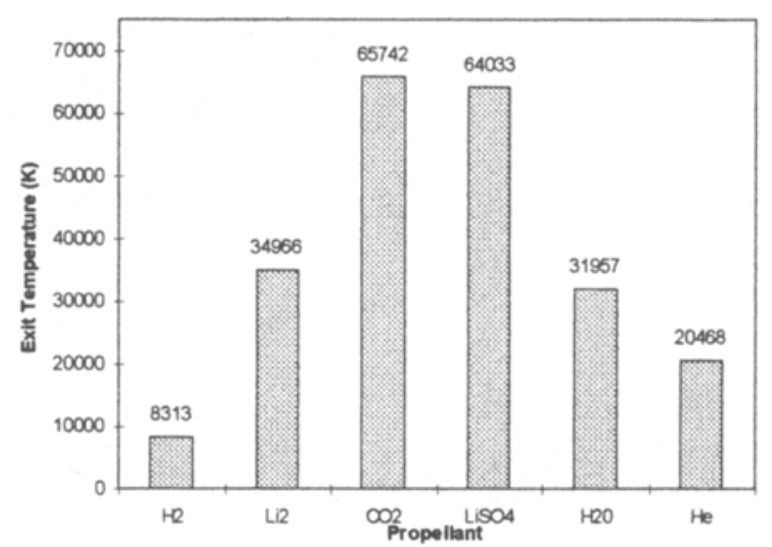

(b) Specific Impulse at $3 \mathrm{~kg} / \mathrm{s}$ Mass Flow Rate.

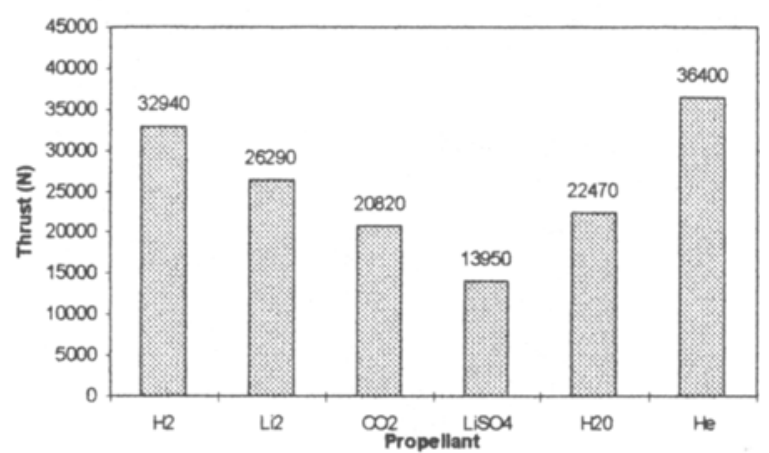

(c) Exit Temperature at $3 \mathrm{~kg} / \mathrm{s}$ Mass Flow Rate.

FIGURE 3. Propulsive Characteristics of Different Propellants.

\section{CONCLUSIONS}

We have presented in this paper an open cycle gas core nuclear rocket that can be energized by controlled fusion reactions which generate a propulsion capability that could open up the solar system and beyond to human exploration. It is based on the simple mirror magnetic confinement concept whose underlying physics has been established by world-wide research on terrestrial fusion power for the past several decades. It is called the gasdynamic mirror because the plasma in it is highly collisional and behaves much like a fluid with confinement properties governed by gasdynamic laws. As a gas core propulsion system the hydrogen propellant is heated primarily by the radiation emitted by the fusion plasma, and when ejected through a nozzle it generates sufficiently large thrust and specific impulse to allow a round trip to Mars to be undertaken in months instead of years. It can be viewed as a near-term device since the confinement physics is quite well understood and the technology for constructing such a system is either currently available or will be so in the near future.

\section{Acknowledgment}

This work was supported by the U.S. National Aeronautics and Space Administration (NASA).

\section{References}

Chandrasekhar, S. (1961) Hydrodynamic and Hydromagnetic Stability, Dover Publications, N.Y..

Diessler, R. G. (1994) Journal of Heat Transfer, May:240-246.

Kammash, T., D. L. Galbraith and T. J. Godfroy (1997) "Asymmetric Gasdynamic Mirror Fusion Propulsion System," AIAA -97-3068, 33rd Joint Propulsion Conference, Seattle, WA

Kammash, T., and M. J. Lee (1995) "Gasdynamic Fusion Propulsion System for Space Exploration," Journal of Propulsion and Power, 11:544-553. 
Kammash, T. (1994) Acta Astronoutica, 34:17.

Mirnov, V. V., and D. D. Ryutov (1979) Sov. Tech. Phys. Letters, 5:279.

Poston, D. I., and T. Kammash (1996) Nuclear Science and Engineering, 122:32.

Ragsdale, R. G., and E. A. Willis Jr. (1971) AIAA-71-641, 7th Propulsion Joint Specialist Conference, Salt Lake City, UT.

\begin{tabular}{|c|c|c|c|}
\hline & Deuterium - Tritium fuel & $\frac{\text { ture }}{\mathrm{R}}$ & \\
\hline D-He ${ }^{3}$ & $\begin{array}{l}\text { Deuterium - Intrum nuel } \\
\text { Deuterium - Helium } 3 \text { fuel }\end{array}$ & $\begin{array}{l}\mathrm{R} \\
\mathrm{R}_{\mathbf{0}}\end{array}$ & $\begin{array}{l}\text { Plasma mirror ratio } \\
\text { Vacuum mirror ratio }\end{array}$ \\
\hline $\mathrm{C}_{\mathrm{p}}$ & Specific Heat & $\mathrm{T}$ & Ion Temperature $(\mathrm{eV})$ \\
\hline$E_{c}$ & Charged Particle Energy Absorbed by & $\mathrm{T}_{\mathrm{H}}$ & Hydrogen Temperature (eV) \\
\hline & Plasma (eV) & $\mathrm{T}_{\mathrm{HO}}$ & Hydrogen Inlet Temperature (eV) \\
\hline$E_{\text {in }}$ & Injection Energy $(\mathrm{eV})$ & $\mathrm{u}$ & Hydrogen Axial Velocity $(\mathrm{m} / \mathrm{s})$ \\
\hline $\mathrm{E}_{\mathrm{L}}$ & Escape Energy (eV) & $v_{\text {th }}$ & Ion Thermal Velocity $(\mathrm{m} / \mathrm{s})$ \\
\hline $\mathrm{f}$ & Fraction to Direct Converter & $\beta$ & Ratio of plasma pressure to magnetic field \\
\hline F & Thrust (N) & & pressure \\
\hline g & Earth's Gravitational Acceleration $\left(\mathrm{m} / \mathrm{s}^{2}\right)$ & $\lambda$ & Collision Mean Free Path (m) \\
\hline$I_{s p}$ & Specific Impulse (s) & $\rho$ & Mass Density $\left(\mathrm{kg} / \mathrm{cm}^{3}\right)$ \\
\hline$l$ & Travel Distance $(\mathrm{km})$ & $\eta_{\mathrm{i}}$ & Injector Efficiency \\
\hline $\mathrm{L}$ & Plasma Length (m) & $\eta_{\mathbf{t}}$ & Thermal Converter Efficiency \\
\hline $\mathrm{m}_{\mathrm{f}}$ & Final (dry) Mass (Mg) & $\eta_{D}$ & Direct Converter Efficiency \\
\hline $\mathbf{n}$ & Number Density $\left(\mathrm{cm}^{-3}\right)$ & $\tau$ & Confinement Time (s) \\
\hline $\mathbf{P}_{\mathrm{B}}$ & Bremsstrahlung Power (MW) & $\tau_{\mathrm{H}}$ & Hydrogen Residence Time (s) \\
\hline $\mathbf{P}_{\mathrm{C}}$ & Charged Particle Power (MW) & $\tau_{R T}$ & Round Trip Travel Time (days) \\
\hline $\mathbf{P}_{\mathrm{f}}$ & Fusion Power (MW) & $<\sigma u\rangle$ & Velocity-Averaged Fusion Cross \\
\hline$P_{r}$ & Radiated Power (MW) & & Section $\left(\mathrm{cm}^{2}\right)$ \\
\hline $\mathbf{P}_{\mathbf{s}}$ & Synchrotron Power (MW) & & \\
\hline $\mathbf{Q}$ & Energy multiplication factor & & \\
\hline
\end{tabular}

